\begin{tabular}{|c|l|}
\hline Title & Top-down effects on antagonistic inducible defense and offense \\
\hline Author(s) & Kishida, Osamu; Trussell, Geoffrey C.; Nishimura, Kinya \\
\hline Citation & Ecology, 90(5), 1217-1226 \\
\hline Issue Date & $2009-05$ \\
\hline Doc URL & http://hdl.handle.net/2115/38515 \\
\hline Type & article \\
\hline File Information & 90-5_p1217-1226.pdf \\
\hline
\end{tabular}

Instructions for use 


\title{
Top-down effects on antagonistic inducible defense and offense
}

\author{
Osamu Kishida, ${ }^{1,2,4}$ Geoffrey C. Trussell, ${ }^{3}$ and Kinya Nishimura ${ }^{1}$ \\ ${ }^{1}$ Graduate School of Fisheries Sciences, Hokkaido University, Hakodate, Hokkaido 041-8611 Japan \\ ${ }^{2}$ Center for Ecological Research, Kyoto University, Otsu, Shiga 520-2113 Japan \\ ${ }^{3}$ Marine Science Center, Northeastern University, 430 Nahant Road, Nahant, Massachusetts 01908 USA
}

\begin{abstract}
Antagonistic phenotypic plasticity may strongly influence trait evolution in tightly interacting predator-prey pairs as well as the role that trait plasticity plays in community dynamics. Most work on trait plasticity has focused on single predator-prey pairs, but prey must often contend with multiple predators in natural environments. Hence, a better understanding of the evolutionary and ecological significance of phenotypic plasticity requires experiments that examine how multiple predators shape prey trait plasticity.

Here, using a simple food chain consisting of a top predator (dragonfly larvae, Aeshna nigroflava), an intermediate predator (salamander larvae, Hynobius retardatus), and frog (Rana pirica) tadpoles as prey, we show that the presence of dragonfly risk cues substantially modifies the intensity of antagonistic morphological plasticity in both amphibians. In the absence of dragonflies, tadpoles produced bulgier bodies in response to salamanders, and salamanders responded to this defense by enlarging their gape size. However, in the presence of dragonfly risk cues, the expression of both antagonistic traits was significantly reduced because tadpoles and salamanders produced phenotypes that are more effective against dragonfly predators. Thus, the reduced antagonism likely emerged, in part, because the benefits of antagonistic trait expression were outweighed by the potential cost of increased vulnerability to dragonfly predation. In addition, our results suggest that when all three species were present, salamander activity levels, which influence the amount of signals required to induce antagonistic traits, were more strongly affected by dragonfly risk cues than were tadpole activity levels. This species-specific difference in activity levels was likely responsible for the reduced tadpole mortality caused by salamanders in the presence vs. absence of dragonfly risk cues. Hence, dragonflies had a positive trait-mediated indirect effect on tadpoles by modifying both the morphological and behavioral traits of salamanders.
\end{abstract}

Key words: arms race; coevolution; gape-limited predation; intraguild predation, morphological change; multiple predators; phenotypic plasticity; predation risk; top predator; trait-mediated indirect interactions; trophic polyphenism.

\section{INTRODUCTION}

Inducible changes in the morphological (Harvell 1984, Lively 1986, Appleton and Palmer 1988, Trussell 1996, Trussell and Smith 2000, Van Buskirk and Schmidt 2000, Relyea 2001, 2003, Trussell and Nicklin 2002) and behavioral (Dill 1987, Lima and Dill 1990, Lima $1998 a, b$, Werner and Peacor 2003) traits of prey in response to cues signaling predation risk are widespread in nature (for review see Tollrian and Harvell 1999). In addition to reducing prey vulnerability to predators, such inducible defenses can strongly influence community dynamics through trait-mediated cascades (Schmitz et al. 1997, 2004, Trussell et al. 2002, 2003, 2006, Van der Stap et al. 2007) or habitat modification (Raimondi et al. 2000).

Manuscript received 3 February 2008; revised 29 July 2008; accepted 3 September 2008. Corresponding Editor: R. K. Zimmer.

${ }^{4}$ Present address: Center for Ecological Research, Kyoto University, Otsu, Shiga 520-2113 Japan.

E-mail: bulgy_tadpoles@hotmail.com
Although much research has documented the ubiquity of inducible defenses in natural systems, two issues have received considerably less attention. First, we are only beginning to understand whether predator species can respond to the inducible defenses of their prey with inducible offenses of their own (Collins and Cheek 1983, Bernays and Chapman 2000, Padilla 2001, Michimae and Hangui 2007). Second, most research on inducible defenses and emerging trait-mediated effects has focused on single predator-prey pairs. This approach has been fruitful but does not address the common ecological reality that prey must often contend with multiple predators (Sih et al. 1998, Relyea 2003, Werner and Peacor 2003). As a result, ecologists have begun to explore how multiple predator effects may shape the inducible defenses of prey, particularly those that are morphologically based (Relyea 2003, Teplitsky et al. 2004, Hoverman and Relyea 2007, Lakowitz et al. 2008). By increasing attention to community-level properties, such as trophic complexity, we will obtain more insight into how ecological context drives the evolution of antagonistic morphological plasticity between predators 
and prey and the importance of trait-mediated indirect interactions to community dynamics (Agrawal 2001, Lima 2002, Werner and Peacor 2003, Miner et al. 2005, Fordyce 2006, Agrawal et al. 2007). For example, while it is well established that predators can initiate strong trait-mediated effects in food webs (Peacor and Werner 2003, Schmitz et al. 2004), little effort has explored whether predator-induced defenses and emergent indirect effects may be modified from the top down by the presence of an additional predator.

The impact of multiple predators on the expression of inducible defenses is likely driven by phenotypic tradeoffs and the ability of prey to recognize cues signaling predation risk, both of which are central to plasticity theory (Via and Lande 1985, Gomulkiewicz and Kirkpatrick 1992, Moran 1992). Phenotypic changes in one species that are induced by another can be directly or indirectly affected in three ways. First, plastic responses may be limited by ecological trade-offs tied to the risk presented by each predator because responses that are efficacious in dealing with one predator may increase the prey's vulnerability to another predator (DeWitt et al. 2000, Benard 2006). Second, in addition to balancing the trade-offs in survivorship associated with each induced phenotype, prey may also experience developmental constraints between phenotypes because of allocation trade-offs or structural conflicts. In general, induced morphological traits are costly to produce and phenotypic integration can impose structural constraints among the traits (Pigliucci and Preston 2004, Relyea 2005). Hence, while prey may be able to produce different phenotypes in response to each predator in isolation, their responses may differ greatly when multiple predators are present and depend on the nature of these constraints and the relative risk presented by each predator.

Finally, prey responses to one predator can be modified if the presence of another predator modifies their interaction intensity. Adaptive phenotypic plasticity requires reliable signals, and many species appear to use signals that are associated with interaction intensity (Wiackowski and Staronska 1999, Van Buskirk and Arioli 2002, Relyea 2004, Schoeppner and Relyea 2008). For example, if a predator-prey pair is sensitive to the presence of a second predator in the system, then the intensity of their interactions and thus the dosage of cues released (by the primary predator) to induce the prey defense may diminish, thereby reducing the degree of inducible defense expression.

We explore these issues in this paper using a simple food chain consisting of a top predator (larvae of the dragonfly Aeshan nigroflava), an intermediate predator (larvae of the salamander Hynobius retardatus), and frog (Rana pirica) tadpoles as prey. We experimentally show that the presence of dragonflies substantially modifies antagonistic plastic interactions between salamanders and tadpoles, as well as their activity levels, and thus transmits a strong trait-mediated indirect effect to tadpoles by modifying salamander foraging success.

\section{Materials And Methods}

Study system and background

Aeshna nigroflava (dragonfly; see Plate 1) are distributed throughout Japan, and their larvae are found primarily in permanent mountain ponds. Hynobius retardatus (salamander; see Plate 1) are endemic to Hokkaido Island (northern island of Japanese archipelago), whereas Rana pirica (frog) are distributed throughout Hokkaido Island and neighboring islands including Sakhalin Island (Russia). Both amphibian larvae are common in mountain ponds and wetlands. On Hokkaido Island, the larvae of all species frequently coexist in mountain ponds. In the lower (salamandertadpole) predator-prey interaction, the larvae of both species exhibit reciprocal morphological changes in each other's presence. Tadpoles ( $R$. pirica) produce bulgy bodies (Fig. 1a) in response to the larval salamander $(H$. retardatus), which is a gape-limited predator that swallows its prey (Kishida and Nishimura 2004). Similarly, high local tadpole density induces salamander larvae to produce a "predaceous phenotype" (Fig. 1b) that is characterized by a large mouth that allows the swallowing of large prey (Michimae and Wakahara 2002). These reciprocal plastic responses are antagonistic because salamander foraging success greatly depends on the balance between their gape size and tadpole body size (Ohdachi 1994, Kishida and Nishimura 2004).

The signals inducing these responses appear to be closely associated with foraging activity. Induction of the bulgy morph in tadpoles requires that they be in close proximity to the salamanders, and increased foraging activity on the part of both amphibians likely increases their degree of contact (Kishida and Nishimura 2004). Induction of the predaceous salamander morphs requires mechanical vibrations from the flapping tails of tadpoles (Michimae et al. 2005). Again such close contact may arise because of high tadpole density or high tadpole foraging activity.

In natural pond habitats, there are several aquatic insects including larval dragonflies (Aeshna nigroflava) that act as top predators. Amphibian larvae commonly alter their behavior (reduced foraging activity) or morphology (high tail fins, small bodies) in response to larval Aeshnid dragonfly risk cues (Van Buskirk and Schmidt 2000, Relyea 2001, Van Buskirk 2002). When dragonflies are present, amphibian larvae having predator-induced phenotypes achieve higher survival rates but slower growth than those without predator-induced phenotypes (Van Buskirk and Relyea 1998). Previous work in our system has shown that both salamanders and tadpoles exhibit behavioral and morphological plasticity in response to chemical risk cues released by dragonflies (Kishida and Nishimura 2005, Iwami et al. 2007). 


\section{Experiments}

We collected frog ( $R$. pirica, $N=5)$ and salamander (H. retardatus, $N=10$ ) egg masses and 30 larval dragonflies (A. nigroflava) from a pond in Akaigawa, Hokkaido, Japan and maintained them in the laboratory at $16^{\circ} \mathrm{C}$. One week after collection, both amphibians began to hatch. Four days after hatching, tadpoles (body length $=5.9 \pm 1.1 \mathrm{~mm}$, mean $\pm \mathrm{SE}, N=20$ ) and salamander (body length $=12.9 \pm 0.7 \mathrm{~mm}, N=20$ ) larvae were randomly selected for use in the experiment.

The experimental units were $22-\mathrm{L}(44 \mathrm{~L} \times 32.5 \mathrm{~W} \times 16$ $\mathrm{cm} \mathrm{D)} \mathrm{aquaria} \mathrm{filled} \mathrm{with} 15 \mathrm{~L}$ of aged tap water. Each aquarium contained a polypropylene colander that housed a single dragonfly. Each colander had a number of slit-like pores (slit size, $1 \times 10 \mathrm{~mm}$; size of colander: top diameter, $8 \mathrm{~cm}$, bottom diameter, $15 \mathrm{~cm}$ ) with a clear cover plate. This design prevented dragonflies from consuming salamanders and tadpoles in each aquarium but allowed the detection of water-borne dragonfly chemical signals by both prey. We established two focal treatments: (1) tadpoles and salamander (40 tadpoles, 1 salamander) and (2) tadpoles, salamander, and dragonfly (40 tadpoles, 1 salamander, 1 dragonfly). In addition, we created four other treatments that facilitated the interpretation of results from the focal treatments: (3) tadpoles only (40 tadpoles), (4) salamander only (1 salamander), (5) tadpoles and dragonfly (40 tadpoles, 1 dragonfly), and (6) salamander and dragonfly (1 salamander, 1 dragonfly). Tadpoles were allowed to forage freely within the aquaria while dragonflies remained isolated within the colander. Each treatment was replicated six times. The initial length of dragonflies used in the experiment was $38.5 \pm 7.9 \mathrm{~mm}$ (mean $\pm \mathrm{SE}$, $N=10)$. The initial densities of tadpoles (279.7 individuals $/ \mathrm{m}^{2}$ ) and salamanders (7.0 individuals $/ \mathrm{m}^{2}$ ) are typical of amphibian hatchling densities in natural ponds (Kishida and Nishimura 2006).

We ran the experiment for 10 days. During this time chironomid larvae $(5 \mathrm{~g}$, frozen stocks were thawed before addition) were spread on the bottom of each aquarium every other day to provide food for tadpoles and salamanders. Tadpoles always fed on chironomids but salamanders fed on either chironomids (salamander only or salamander and dragonfly treatments) or chironomids and tadpoles (tadpole and salamander treatment). Dragonflies were fed $150 \mathrm{mg}$ of tadpoles every other day. To maintain adequate water quality, 5 $\mathrm{L}$ of the water in all aquaria was changed every second day throughout the experiment. Although we fed dragonflies tadpoles, previous work has shown that risk cues from either injured or consumed tadpoles do not induce behavioral or morphological defenses in both tadpoles and salamanders (Iwami et al. 2007; O. Kishida, T. Iwami, and K. Nishimura, unpublished data). Hence, dragonfly chemical risk cues, when present, were responsible for inducing or modifying the plasticity we observed in both amphibian larvae.

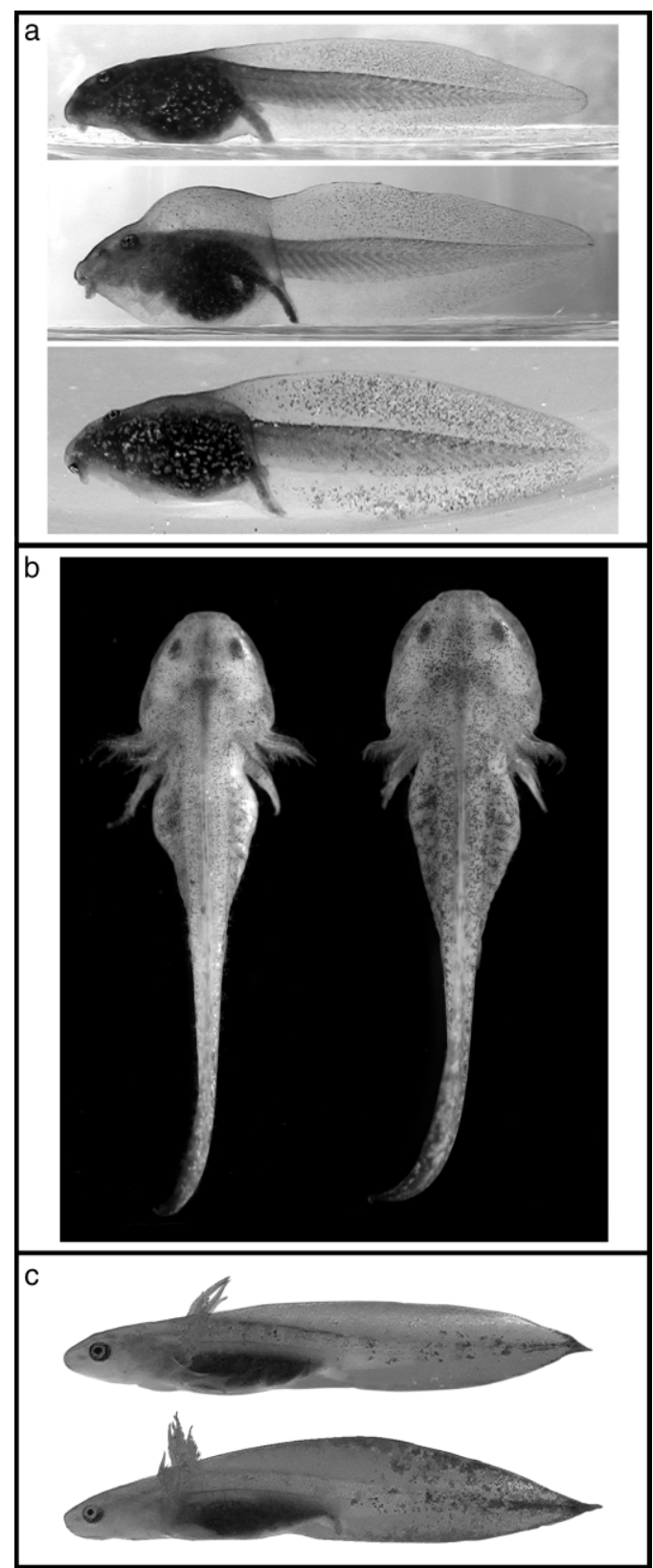

FIG. 1. Photographs of morphological plasticity in (a) Rana pirica frog tadpoles (upper, non-induced basic morph; middle, salamander-induced bulgy morph; bottom, dragonfly-induced high-tail morph); (b) Hynobius retardatus salamander larvae (left, non-induced basic morph; right, tadpole-induced predaceous morph); (c) Hynobius retardatus salamander larvae (upper, non-induced basic morph; bottom, dragonfly-induced high-tail morph).

We measured tadpole and salamander activity 10 times over two days of the experiment. Observations were conducted at hourly intervals from 10:00 to 15:00 on days 8 and 9 of the experiment. To measure tadpole activity during each observation, we recorded the activity (moving or stationary) of 10 randomly selected 
tadpoles in aquaria containing the four tadpole treatments. Activity was expressed as the proportion of tadpoles moving over the 10 observation periods. To measure salamander activity, we followed each individual salamander for 30 seconds during each observation period to determine if any movement occurred. Salamanders were scored as moving regardless of the amount of time spent moving and the number of movements during each observation. Hence, movement score of each salamander was represented as a proportion relative to total observation times (i.e., proportion of movement occurrence $=x_{i} / 10$, where $x_{i}$ represents movement score of individual $i$ ). This scoring method is appropriate for quantifying salamander activity because they typically display short and infrequent bursts of activity. At the end of the experiment, all surviving tadpoles were counted to determine survival rates that were arcsine-transformed to meet the assumptions of statistical analyses. There was no salamander mortality.

To characterize salamander phenotypes (i.e., tadpoleinduced predaceous phenotype and dragonfly-induced high-tail phenotype), we measured four traits: head width at eye level, largest head width, body length (snout-vent length), and maximum tail depth. The ratio of head width at eye level to the largest head width reflects the magnitude of predaceous phenotype expression (Michimae and Wakahara 2001). Increased salamander tail depth is indicative of the dragonfly-induced phenotype (Fig. 1c), and this trait can be influenced by salamander size. We used body length as the canonical size measurement because it is relatively stable whereas other traits also change when the predaceous phenotype is induced. In our experiment, we detected no covariance between tail depth and body length within each treatment (all $P \geq 0.27$ ). Hence, we calculated relative tail depth (tail depth/body length) as an index of tail induction for the one salamander in each aquarium.

To characterize tadpole phenotypes (i.e., salamanderinduced bulgy phenotype, dragonfly-induced high-tail phenotype) 16 surviving tadpoles were randomly selected from each aquarium and measured for three traits: body length (snout-vent length), maximum body depth, and maximum tail depth. Size-adjusted body depth and tail depth are diagnostic of the predator-specific morphologies. Relative to non-induced phenotypes, the salamander-specific tadpole is characterized by a higher tail and a bulgy body (increased body depth) whereas the dragonfly-specific tadpole is characterized by just higher tail depth (Kishida and Nishimura 2005). Because these traits can covary with tadpole size (body length), we used analyses of covariance (ANCOVA) to adjust for this effect. ANCOVA revealed that the slopes of regressions for both tadpole body depth $\left(F_{1,355}=\right.$ $0.01, P=0.93)$ and tail depth $\left(F_{1,355}=0.17, P=0.68\right)$ as a function of body length were equal for all treatments. Hence, we were able to compare size-adjusted mean body depth and tail depth because the scaling between these focal traits and size was the same across our experimental treatments. Because we had multiple tadpoles within each aquarium that were not independent, replicate aquaria were considered a random factor nested within our experimental treatments.

In the tadpole-salamander and tadpole-salamanderdragonfly treatments, salamanders consumed tadpoles throughout the experiment. Salamanders consumed 9.7 \pm 4.5 tadpoles (mean $\pm \mathrm{SE}$ ) in the tadpole-salamander treatment and $5.7 \pm 1.2$ tadpoles in the tadpolesalamander-dragonfly treatment (see Results). To exclude the possibility that selective predation on noninduced phenotypes exaggerated the apparent extent of inducible defense expression in our treatments, we corrected the mortality data before analysis (see Appendix). After correction, resulting aquarium means were used in statistical analyses.

\section{Statistical analyses}

We conducted separate two-way ANCOVAs or ANOVAs to examine the induction of antagonistic plastic morphologies and how these responses were influenced by the presence of dragonflies as a top predator. A two-way ANCOVA that considered salamander (present, absent) and dragonfly treatments (present, absent) as fixed effects and body length as a covariate was used to evaluate bulginess expression in tadpoles. A similar ANCOVA model was used to evaluate treatment effects on tadpole tail depth. A two-way ANOVA that considered tadpole (present, absent) and dragonfly (present, absent) treatments as fixed effects evaluated the degree of predaceous phenotype expression and tail depth change in salamanders. Two-way ANOVAs, assuming multiplicative risk (Wilbur and Fauth 1990, Wootton 1994, Sih et al. 1998), were also used to examine induced predator effects on tadpole and salamander activity levels. Hence, activity data were $\log _{10}$-transformed before analysis. In addition, we were also explicitly interested in determining whether salamanders vs. tadpoles were more sensitive to dragonfly risk cues when all three species were interacting. Hence, we compared the activity levels of both amphibians between the tadpole-salamander-dragonfly treatment and the tadpole-salamander treatment with MANOVA. We used this analysis because tadpole and salamander activity levels are likely not independent. We focused on the interaction between amphibian (tadpoles and salamanders) activity and treatment (dragonfly presence or absence) to determine which amphibian was more sensitive to dragonfly risk cues.

Because our analyses sometimes yielded significant interactions, post hoc comparisons (Tukey's hsd) were used to identify those treatments that were significantly different from one another. Finally, because our analyses consistently demonstrated an effect of dragonflies on salamander and tadpole traits that determine how intensely they interact, we also examined the effect of dragonflies on tadpole mortality caused by salamander predation with a $t$ test. All analyses were performed 

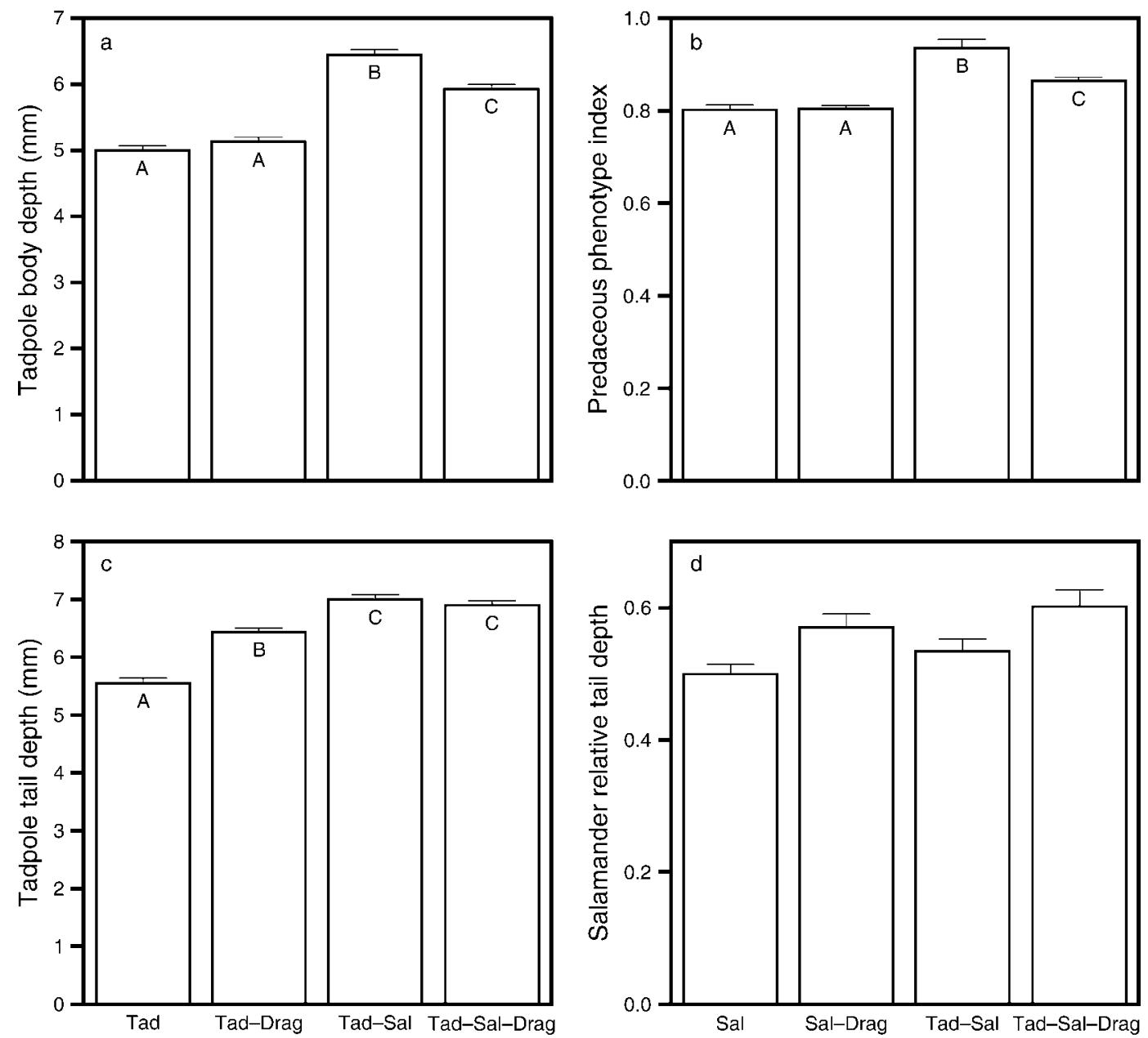

FIG. 2. Morphological traits of tadpoles (R. pirica) and salamanders (H. retardatus) raised under different experimental combinations: (a) tadpole body depth, (b) index of salamander predaceous phenotype, (c) tadpole tail depth, and (d) salamander tail depth. "Tad," "Drag," and "Sal" are abbreviations of tadpole, dragonfly, and salamander, respectively. Error bars denote one standard error ( $N=6$ replicates per treatment). When necessary, post hoc comparisons (Tukey's hsd tests) were performed to resolve differences among experimental treatments. Treatment means sharing the same uppercase letter are not significantly different from one another $(P<0.05)$. In panel $(\mathrm{d})$, interaction terms were not significant in two-way ANOVA, so post hoc tests were not conducted.

using JMP software (SAS Institute, Cary, North Carolina, USA), which uses the Satterthwaite approximation to calculate degrees of freedom when models include nested terms (i.e., replicate aquaria).

\section{RESUlts}

We found a highly significant interaction $\left(F_{1,20}=\right.$ 39.16, $P<0.0001)$ between the effects of salamander presence $\left(F_{1,21}=389.32, P<0.0001\right)$ and dragonfly presence $\left(F_{1,21}=13.81, P=0.0013\right.$; Fig. 2a $)$ on tadpole body depth. Tadpoles produced significantly bulgier bodies in the presence vs. absence of salamanders. However, the significant interaction between the effects of salamander presence and dragonfly presence on tadpole body depth revealed that the induction of bulgier bodies by salamanders was stronger when dragonflies were absent. In the absence of salamanders, we did not detect a significant effect of dragonflies on tadpole bulginess (Tukey's hsd, $P=0.398$ ).

Similar to our results for tadpoles, induction of the predaceous morph (larger mouth) in salamanders was strongly shaped by an interaction $\left(F_{1,20}=10.47, P=\right.$ $0.0041)$ between the presence and absence of tadpoles $\left(F_{1,20}=68.38, P<0.0001\right)$ and dragonflies $\left(F_{1,20}=8.69\right.$, $P=0.0079$; Fig. 2b). Salamanders produced larger mouths in the presence of tadpoles but the significant interaction indicated that this effect was not as strong when dragonflies were present. In the absence of tadpoles, dragonflies had no effect on predaceous morph expression (Tukey's hsd, $P=0.9970$ ).

The presence of salamanders $\left(F_{1,21}=159.05, P<\right.$ $0.0001)$ and dragonflies $\left(F_{1,21}=25.64, P<0.0001\right)$ induced deeper tails in tadpoles, and a significant interaction $\left(F_{1,20}=42.36, P<0.0001\right)$ revealed that 

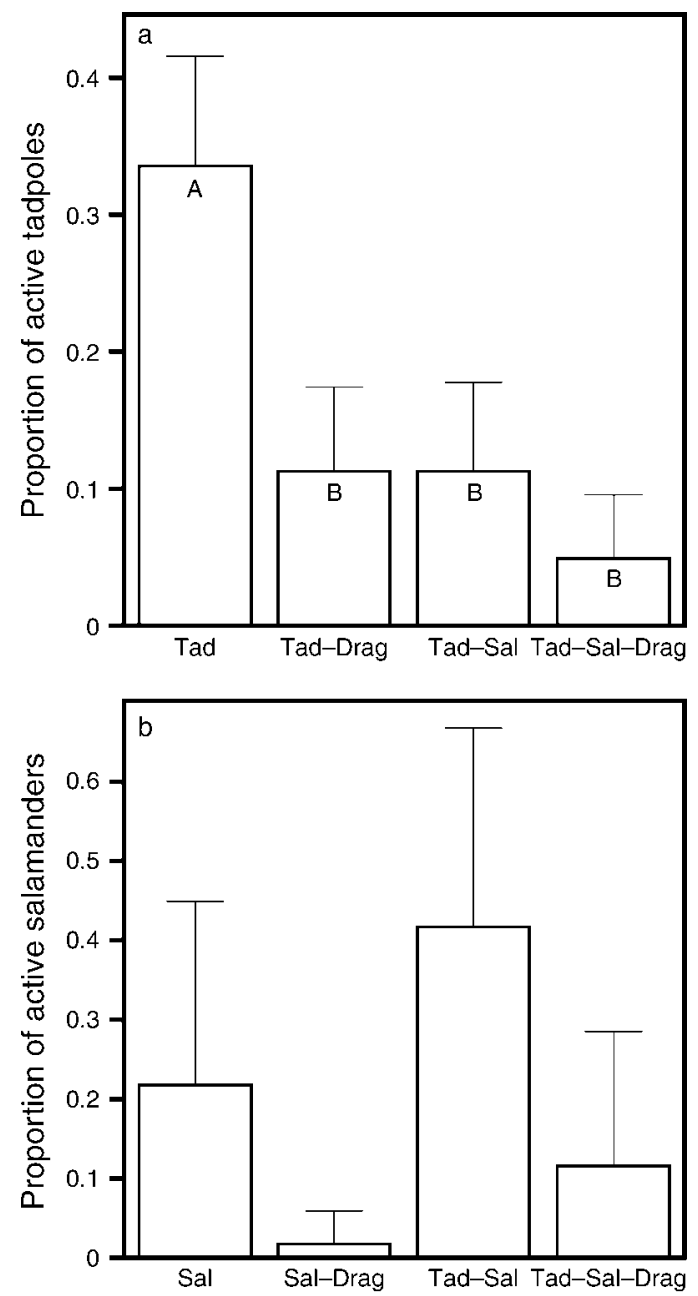

FIG. 3. The activity level of tadpoles (R. pirica) and salamanders ( $H$. retardatus): (a) proportion of active tadpoles and (b) proportion of salamanders exhibiting movement. Abbreviations are as in Fig. 1. Error bars denote 95\% confidence intervals $(N=6$ replicates per treatment). When necessary, post hoc comparisons (Tukey's hsd tests) were performed to resolve differences among experimental treatments. Treatment means sharing the same uppercase letter are not significantly different from one another $(P<0.05)$. In panel (b), interaction terms were not significant in two-way ANOVA, so post hoc tests were not conducted.

the effect of salamanders on tadpole tail depth was stronger in the absence of dragonflies (Fig. 2c). For salamanders, only the presence of dragonflies $\left(F_{1,20}=\right.$ $11.91, P=0.0025)$ induced the production of deeper tails; neither the effect of tadpoles $\left(F_{1,20}=2.70, P=\right.$ $0.1159)$ nor the interaction $\left(F_{1,20}=0.01, P=0.9353\right)$ was significant (Fig. 2d).

The presence of salamanders $\left(F_{1,20}=33.06, P<\right.$ $0.0001)$ and dragonflies $\left(F_{1,20}=33.99, P<0.0001\right)$ caused reductions in tadpole activity but a significant interaction indicated that the presence of both predators did not further diminish tadpole activity levels $\left(F_{1,20}=\right.$ 8.50, $P=0.009$; Fig. 3a). For salamanders, both the absence of tadpoles $\left(F_{1,20}=4.38, P=0.049\right)$ and the presence of dragonflies $\left(F_{1,20}=11.90, P=0.003\right)$ significantly reduced their activity and these effects were additive (interaction: $F_{1,20}=0.35, P=0.561$; Fig. 3b). MANOVA also revealed tadpoles and salamanders were less active in the presence of dragonflies compared to when they were together alone $\left(F_{1,10}=8.10, P=0.017\right)$. Moreover, a significant interaction $\left(F_{1,10}=4.54, P=\right.$ $0.059)$ strongly suggested that salamanders were more sensitive to dragonfly risk cues than were tadpoles.

Tadpole mortality (mean \pm SE) was very low in the tadpole $(1.5 \% \pm 0.01 \%)$ and tadpole-dragonfly treatments $(0.01 \% \pm 0.01 \%)$. In contrast, salamanders caused tadpole mortality to be considerably higher in the tadpole-salamander $(24.3 \% \pm 0.05 \%)$ and the tadpolesalamander-dragonfly treatments $(14.5 \% \pm 0.01 \%)$. Moreover, tadpole mortality was significantly lower $\left(t_{10}=2.17, P=0.027\right.$; Fig. 4$)$ when dragonflies were also present suggesting that dragonflies diminished the impact of salamanders on tadpole survivorship.

\section{Discussion}

Although antagonistic inducible responses are well known in plant-herbivore interactions (for review see Karban and Baldwin 1997), we are just beginning to explore their evolutionary and ecological significance in other predator-prey pairs (Bernays and Chapman 2000, Padilla 2001, Kopp and Tollrian 2003, Kishida et al. 2006). In particular, we have a limited understanding of how ecological context shapes these interactions (Agrawal et al. 2007). Here we have shown that the antagonistic expression of inducible defenses in tadpoles and inducible offenses in salamanders is strongly reduced by the presence of dragonfly risk cues. In the absence of dragonflies, salamanders induced tadpoles to produce bulgier bodies compared to those without salamander cues (Fig. 2a), and salamanders responded to defended prey by producing predaceous phenotypes having larger mouths (Fig. 2b). However, in the presence of dragonfly risk cues, the expression of both

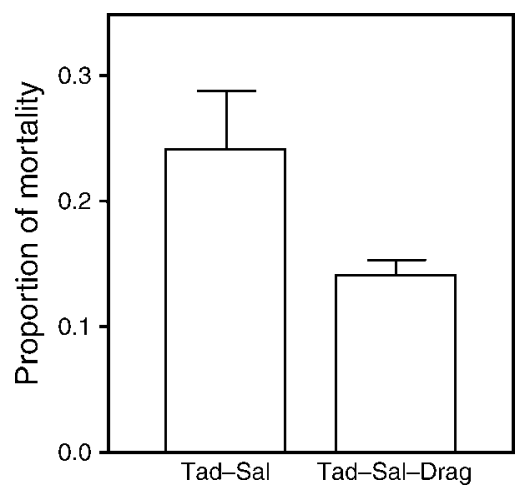

FIG. 4. Tadpole (R. pirica) mortality rates in the tadpolesalamander and tadpole-salamander-dragonfly treatments. Error bars denote standard error $(N=6$ replicates per treatment). No two-way ANOVA was conducted. 


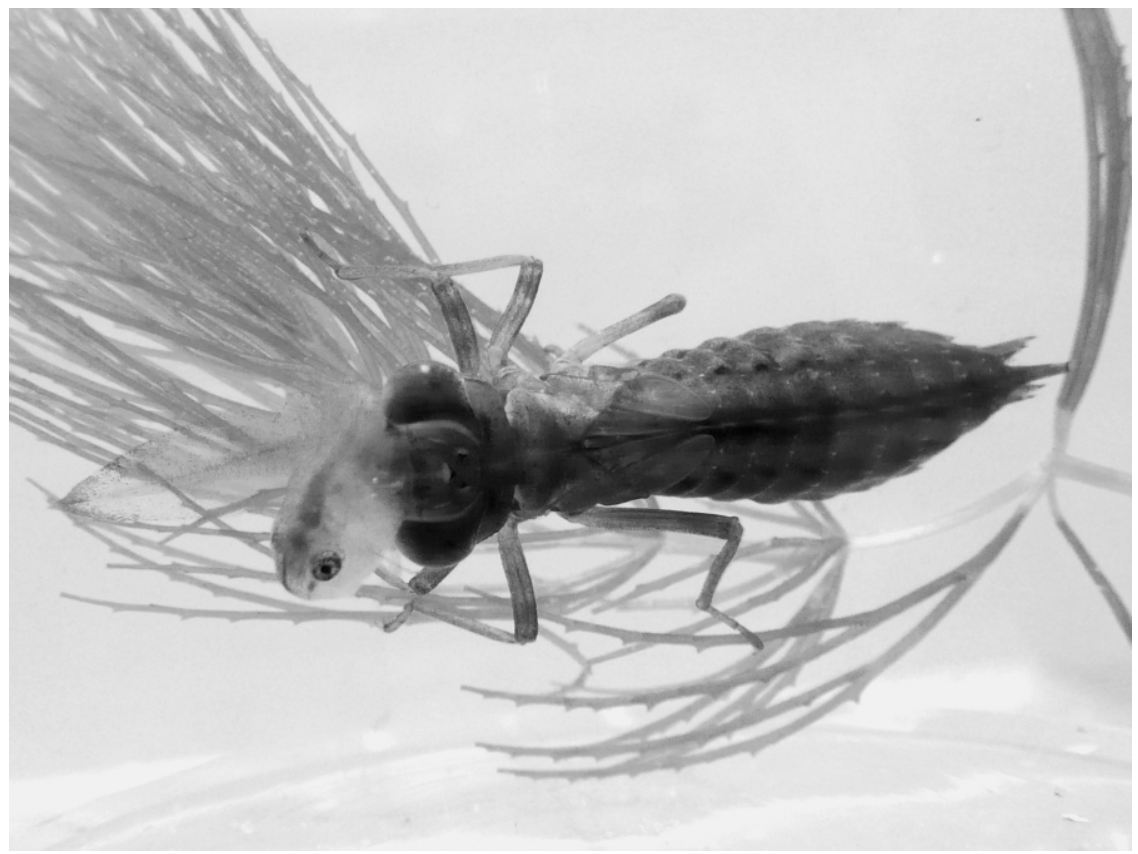

Plate 1. Top-predator dragonfly larva (Aeshna nigroflava) consuming intermediate-predator salamander larva (Hynobius retardatus). Photo credit: O. Kishida.

amphibian traits was significantly reduced. In the presence of dragonflies, tadpoles exposed to salamanders still produced significantly bulgier bodies relative to the no-predator control (tadpoles only), but bulginess expression was reduced by $8.6 \%$ compared to when tadpoles were exposed to only salamanders. Similarly, salamanders with tadpoles and dragonflies also produced significantly wider mouths relative to their control (no tadpoles present) but expression of the predaceous phenotype was reduced by $8.5 \%$ compared to when dragonfly cues were absent.

The dragonfly-induced reduction in the antagonistic plasticity between salamanders and tadpoles is likely adaptive in reducing the vulnerability of both amphibians to dragonfly predators. Previous work (Kishida and Nishimura 2005) found that the survival rate of bulgy tadpoles (induced by salamanders) is lower than the deeper tail phenotypes (induced by dragonflies) when confronted with dragonfly predation. In contrast, they found no differences in survivorship between bulgy and non-induced tadpoles when both phenotypes were offered to dragonflies. Finally, tadpoles having deeper tails were less vulnerable to salamander predation than non-induced tadpoles. These results suggest that while each tadpole phenotype has superior benefits in environments that contain only the inducing predator, deeptailed phenotypes are better adapted than alternative phenotypes (non-induced and bulgy) in environments having both salamander and dragonfly predators. Similar reasoning also explains the dragonfly-induced reduction in predaceous phenotype expression in salamanders because the slower burst swimming of the predaceous phenotype makes it more susceptible to dragonfly predation than the non-predaceous phenotype (G. Miyazaki, unpublished data).

Our results indicate that the benefits of antagonistic phenotype expression are outweighed by the potential cost of increased vulnerability to dragonfly predation. Although allocation trade-offs or developmental conflicts may constrain the induction of multiple phenotypes (Pigliucci and Preston 2004, Relyea 2005) and thus underlie the observed reduction in antagonistic plasticity, we found little evidence of such trade-offs. Indeed, in tadpoles there was no negative correlation between the expression of deeper tails and bulgier bodies (Fig. 2a, c). For example, tadpoles in the tadpole-salamander and the tadpole-salamander-dragonfly treatments differed considerably in bulginess but had statistically identical tail depths. Moreover, linear regressions of tadpole bulginess as a function of tail depth for each treatment combination yielded no significant negative relationships $(0.10 \leq P \leq 0.73)$.

For salamanders, we did not find abundant evidence of allocation trade-offs between predaceous phenotype and deeper tail trait expression in three of our four treatments $(0.38 \leq P \leq 0.56)$. However, in the salamander-dragonfly treatment, these traits were negatively correlated $\left(R^{2}=0.79, P=0.011\right)$, with salamanders having smaller mouths as tail depth increased. This result suggests that the cost of increased vulnerability to dragonfly predation outweighs that associated with reduced predaceous phenotype expression. Hence, the emergence of allocation or developmental constraints may be tightly linked to the 
vulnerability of salamanders to predation risk. In contrast, we detected no trade-off between these traits in the tadpole-salamander-dragonfly treatment $(P=$ $0.56)$, which suggests that the benefits of successful foraging may offset the costs of increased vulnerability to dragonflies. Indeed, comparison of these two treatments found that, for a given relative tail depth, salamanders in the tadpole-salamander-dragonfly treatment had a predaceous index that was $7.5 \%$ greater than that of salamanders in the salamander-dragonfly treatment (ANCOVA: $F_{1,8}=62.36, P<0.0001$ ).

The reduced antagonism between tadpoles and salamanders may have emerged because of reductions in the quantity of induction signals caused by the presence of dragonflies. The signals inducing the bulgy phenotype in tadpoles require close proximity with salamanders (Kishida and Nishimura 2004), and the same is true for salamanders because induction of the predaceous phenotype requires vibrations caused by the flapping tails of tadpoles (Michimae et al. 2005). These signals are obviously influenced by the behavioral activity of both amphibians, and increased activity should enhance the degree of antagonistic trait expression. In our experiment, salamanders were most active in the tadpole-salamander treatment (Fig. 3b), where we observed the bulgiest bodies in tadpoles and the widest mouth in salamanders. However, the addition of dragonflies caused a drastic reduction in salamander activity level and, as a result, tadpole bulginess and salamander mouth width was reduced. These results suggest that the top-down effects of dragonflies on salamanders is likely responsible for reductions in signal transmission because tadpole activity was similarly reduced in the tadpole-salamander and tadpole-salamander-dragonfly treatments (Fig. 3a). If tadpole activity levels were driving the antagonistic plasticity from the bottom up, then one would expect tadpole bulginess and predaceous phenotype expression of salamanders to be similar between the tadpole-salamander and tadpole-salamander-dragonfly treatments.

Previous studies on inducible defenses in the presence of multiple predators found that prey exhibited defenses that were either intermediate or similar to those expressed in response to the riskier predator alone (Relyea 2003, Teplitsky et al. 2004, Hoverman and Relyea 2007). These studies did not explicitly address potential interactions between predators, because predators were fed separate prey items and were segregated from one another in experimental enclosures. Thus, the changes in the expression of inducible defenses in these studies were not tied to how additional predator presence modified the intensity of interactions between a focal predator-prey pair (i.e., tadpoles and salamanders). As we have shown here, such interaction modifications can dictate which traits are produced. Hence, our study highlights the importance of examining how direct and indirect interactions between multiple predators and their prey can shape trait plasticity.

The top-down effect of dragonflies on tadpolesalamander antagonistic plasticity and activity levels also strongly influenced tadpole survivorship (Fig. 4). Tadpole mortality was significantly higher in the tadpole-salamander treatment than in the tadpolesalamander-dragonfly treatment. As discussed before, compared to the tadpole-salamander treatment, the addition of dragonflies induced reduced activity levels $(71 \%)$ and predaceous phenotype expression $(8.5 \%)$ in salamanders (Figs. 2b and $3 \mathrm{~b}$ ). Indeed, our results also suggest that salamanders are more sensitive to dragonfly risk cues when all three species interact. As a result, salamanders experienced reduced foraging success despite having access to tadpoles that were $8.4 \%$ less bulgy than those in the tadpole-salamander treatment (Fig. 2a). We suggest that the reduced foraging success of salamanders in this treatment was more likely driven by their reduced activity in response to dragonfly risk cues rather than their inability to ingest the available tadpole prey.

Our results also suggest that trait plasticity may play an important role in intraguild predator-prey dynamics. Although intraguild predation is ubiquitous in natural systems (Polis et al. 1989, Polis and Holt 1992, Arim and Marquet 2004), theory predicts that intraguild predation can have destabilizing effects that prevent its persistence in ecological time (Holt and Polis 1997, Tanabe and Namba 2005, Rosenheim 2007). Recent mathematical models (Okuyama and Ruyle 2003), however, suggest that adaptive phenotypic plasticity may prevent such destabilization because inducible defenses in intermediate predators (i.e., intraguild prey) maintain their density while reducing their consumption of basal prey, thus relaxing how intensely they compete with top predators (i.e., intraguild predator; Arim and Marquet 2004). In our study, salamanders (intermediate predator) reduced both their activity levels and offensive morphology in response to dragonfly (top predator) predation risk. Both of these responses will increase their survivorship and reduce salamander impacts on tadpoles (basal prey), which may allow intraguild predation to persist. Evidence of such positive, indirect top-predator effects on basal prey that are mediated by the adaptive plasticity in intermediate predators in response to top predators is mounting (Soluk 1993, Okuyama 2002, Rudolf 2006).

A rapidly growing literature has highlighted the importance of consumptive and nonconsumptive predator effects on prey populations and the emergence of density and trait-mediated indirect interactions (Werner and Peacor 2003, Schmitz et al. 2004). Here we have shown that the presence of a top predator (dragonfly) can strongly alter the nonconsumptive and consumptive effects of a secondary predator (salamander) on its prey. It is clear that the trait-mediated effects of dragonflies in this system can be quite strong and are likely similar in 
sign and strength to their density-mediated effects. Future work in this and other systems that more fully capture the complexity of natural food webs will provide critical insight into how ecological context can shape the importance of trait and density-mediated effects to the evolution of predator-prey interactions and community dynamics (Miner et al. 2005, Ohgushi 2005, Agrawal et al. 2007).

\section{ACKNOWLEDGMents}

We thank Tadashi Iwami, Gen Miyazaki, and Nao Sugawara for the significant comments on the early draft of manuscript. We also thank Takuya Ikawa for discussions about phenotypic plasticity. We are very grateful to three anonymous reviewers for their constructive comments on the manuscript. This work was supported by a Grant-in-Aid for Scientific Research (number 16370008) to K. Nishimura from the Ministry of Education, Culture, Sports, Science, and Technology, and in part by a Grant-in-Aid for a Research Fellow of the Japan Society for the Promotion of Science for young scientists (number 09035) to O. Kishida and a grant from the U.S. National Science Foundation (OCE-0727628) to G. C. Trussell.

\section{Literature Cited}

Agrawal, A. A. 2001. Phenotypic plasticity in the interactions and evolution of species. Science 294:321-326.

Agrawal, A. A., et al. 2007. Filling key gaps in population and community ecology. Frontiers in Ecology and the Environment 5:145-152.

Appleton, R. D., and R. A. Palmer. 1988. Water-borne stimuli released by predatory crabs and damaged prey induce more predator-resistant shells in a marine gastropod. Proceedings of the National Academy of Sciences (USA) 85:4387-4391.

Arim, M., and P. A. Marquet. 2004. Intraguild predation: a widespread interaction related to species biology. Ecology Letters 7:557-564.

Benard, M. F. 2006. Survival trade-offs between two predatorinduced phenotypes in Pacific treefrogs (Pseudacris regilla). Ecology 87:340-346.

Bernays, E. A., and R. F. Chapman. 2000. Plant secondary compounds and grasshoppers: beyond plant defenses. Journal of Chemical Ecology 26:1773-1794.

Collins, J. P., and J. E. Cheek. 1983. Effect of food and density on development of typical and cannibalistic salamander larvae in Ambystoma tigrinum nebulosum. American Zoologist 23:77-84.

DeWitt, T. J., B. W. Robinson, and D. S. Wilson. 2000. Functional diversity among predators of a freshwater snail imposes an adaptive trade-off for shell morphology. Evolutionary Ecology Research 2:129-148.

Dill, L. M. 1987. Animal decision making and its ecological consequences: the future of aquatic ecology and behavior. Canadian Journal of Zoology 65:803-811.

Fordyce, J. A. 2006. The evolutionary consequences of ecological interactions mediated through phenotypic plasticity. Journal of Experimental Biology 209:2377-2383.

Gomulkiewicz, R., and M. Kirkpatrick. 1992. Quantitative genetics and the evolution of reaction norms. Evolution 46: 390-411.

Harvell, C. D. 1984. Predator-induced defense in a marine bryozoan. Science 224:1357-1359.

Holt, R. D., and G. A. Polis. 1997. A theoretical framework for intraguild predation. American Naturalist 149:745-765.

Hoverman, J. T., and R. A. Relyea. 2007. The rules of engagement: how to defend against combinations of predators. Oecologia 154:551-560.

Iwami, T., O. Kishida, and K. Nishimura. 2007. Direct and indirect induction of a compensatory phenotype that alleviates the costs of an inducible defense. PLoS ONE 2: e1084.

Karban, R., and I. T. Baldwin. 1997. Induced responses to herbivory. University of Chicago Press, Chicago, Illinois, USA.

Kishida, O., Y. Mizuta, and K. Nishimura. 2006. Reciprocal phenotypic plasticity in a predator-prey interaction between larval amphibians. Ecology 87:1599-1604.

Kishida, O., and K. Nishimura. 2004. Bulgy tadpoles: inducible defense morph. Oecologia 140:414-421.

Kishida, O., and K. Nishimura. 2005. Multiple inducible defenses against multiple predators in anuran tadpoles (Rana pirica). Evolutionary Ecology Research 7:619-631.

Kishida, O., and K. Nishimura. 2006. Flexible architecture of inducible morphological plasticity. Journal of Animal Ecology 75:705-712.

Kopp, M., and R. Tollrian. 2003. Reciprocal phenotypic plasticity in a predator-prey system: inducible offences against inducible defences? Ecology Letters 6:742-748.

Lakowitz, T., C. Bronmark, and P. Nystrom. 2008. Tuning in to multiple predators: conflicting demands for shell morphology in a freshwater snail. Freshwater Biology 53:2184 2191.

Lima, S. L. 1998a. Nonlethal effects in the ecology of predatorprey interactions. BioScience 48:25-34.

Lima, S. L. 1998b. Stress and decision making under the risk of predation: recent developments from behavioral, reproductive, and ecological perspectives. Advances in the Study of Behavior 28:215-290.

Lima, S. L. 2002. Putting predators back into behavioral predator-prey interactions. Trends in Ecology and Evolution 17:70-75.

Lima, S. L., and L. M. Dill. 1990. Behavioral decisions made under the risk of predation: a review and prospectus. Canadian Journal of Zoology 68:619-640.

Lively, C. M. 1986. Predator-induced shell dimorphism in the acorn barnacle Chthamalus anisopoma. Evolution 67:858864.

Michimae, H., and J. Hangui. 2007. A trade-off between prey- and predator-induced polyphenisms in larvae of the salamander Hynobius retardatus. Behavioral Ecology and Sociobiology 62:699-704.

Michimae, H., K. Nishimura, and M. Wakahara. 2005. Mechanical vibrations from tadpoles' flapping tails transform salamander's carnivorous morphology. Biology Letters 1:75-77.

Michimae, H., and M. Wakahara. 2001. Factors which affect the occurrence of cannibalism and the broad-headed "cannibal" morph in larvae of the salamander Hynobius retardatus. Behavioral Ecology and Sociobiology 50:339-345.

Michimae, H., and M. Wakahara. 2002. A tadpole-induced polyphenism in the salamander Hynobius retardatus. Evolution 56:2029-2038.

Miner, B. G., S. E. Sultan, S. G. Morgan, D. K. Padilla, and R. A. Relyea. 2005. Ecological consequences of phenotypic plasticity. Trends in Ecology and Evolution 20:685-692.

Moran, N. 1992. The evolutionary maintenance of alternative phenotypes. American Naturalist 139:971-989.

Ohdachi, S. 1994. Growth, metamorphosis, and gape-limited cannibalism and predation on tadpoles in larvae of salamanders Hynobius retardatus. Zoological Science 11:127-131.

Ohgushi, T. 2005. Indirect interaction webs: herbivore-induced effects through trait change in plants. Annual Review of Ecology, Evolution, and Systematics 36:81-105.

Okuyama, T. 2002. The role of antipredator behavior in an experimental community of jumping spiders with intraguild predation. Population Ecology 44:121-125.

Okuyama, T., and R. L. Ruyle. 2003. Analysis of adaptive foraging in an intraguild predation system. Web Ecology 4: $1-6$. 
Padilla, D. K. 2001. Food and environmental cues trigger an inducible offence. Evolutionary Ecology Research 3:15-25.

Pigliucci, M., and K. Preston. 2004. Phenotypic integration: studying the ecology and evolution of complex phenotypes. Oxford University Press, New York, New York, USA.

Polis, G. A., and R. D. Holt. 1992. Intraguild predation: the dynamics of complex trophic interactions. Trends in Ecology and Evolution 7:151-154.

Polis, G. A., C. A. Myers, and R. D. Holt. 1989. The ecology and evolution of intraguild predation: potential competitors that eat each other. Annual Review of Ecology and Systematics 20:297-330.

Raimondi, P. T., S. E. Forde, L. F. Delph, and C. M. Lively. 2000. Processes structuring communities: evidence for traitmediated indirect effects through induced polymorphisms. Oikos 91:353-361.

Relyea, R. A. 2001. Morphological and behavioral plasticity of larval anurans in response to different predators. Ecology 82: 523-540.

Relyea, R. A. 2003. How prey respond to combined predators: a review and an empirical test. Ecology 84:1827-1839.

Relyea, R. A. 2004. Fine-tuned phenotypes: tadpole plasticity under 16 combinations of predators and competitors. Ecology 85:172-179.

Relyea, R. A. 2005. Constraints on inducible defenses. Pages 189-207 in P. Barbosa and I. Castellanos, editors. Ecology of predator-prey interactions. Oxford University Press, New York, New York, USA.

Rosenheim, J. A. 2007. Intraguild predation: new theoretical and empirical perspectives. Ecology 88:2679-2680.

Rudolf, V. 2006. The influence of size-specific indirect interactions in predator-prey systems. Ecology 87:362-371.

Schmitz, O. J., A. P. Beckerman, and K. M. O'Brien. 1997. Behaviorally mediated trophic cascades: effects of predation risk on food web interactions. Ecology 78:1388-1399.

Schmitz, O. J., V. Krivan, and O. Ovadia. 2004. Trophic cascades: the primacy of trait-mediated indirect interactions. Ecology Letters 7:153-163.

Schoeppner, N. M., and R. A. Relyea. 2008. Detecting small environmental differences: risk-response curves for predatorinduced behavior and morphology. Oecologia 154:743-754.

Sih, A., G. Englund, and D. Wooster. 1998. Emergent impacts of multiple predators on prey. Trends in Ecology and Evolution 13:350-355.

Soluk, D. A. 1993. Multiple predator effects: predicting combined functional response of stream fish and invertebrate predators. Ecology 74:219-225.

Tanabe, K., and T. Namba. 2005. Omnivory creates chaos in simple food web models. Ecology 86:3411-3414.

Teplitsky, C., S. Plenet, and P. Joly. 2004. Hierarchical responses of tadpoles to multiple predators. Ecology 85: $2888-2894$.
Tollrian, R., and C. D. Harvell. 1999. The ecology and evolution of inducible defenses. Princeton University Press, Princeton, New Jersey, USA.

Trussell, G. C. 1996. Phenotypic plasticity in an intertidal snail: the role of a common crab predator. Evolution 50:448-454.

Trussell, G. C., P. J. Ewanchuk, and M. D. Bertness. 2002. Field evidence of trait-mediated indirect interactions in a rocky intertidal food web. Ecology Letters 5:241-245.

Trussell, G. C., P. J. Ewanchuk, and M. D. Bertness. 2003. Traitmediated effects in rocky intertidal food chains: predator risk cues alter prey feeding rates. Ecology 84:629-640.

Trussell, G. C., P. J. Ewanchuk, and C. M. Matassa. 2006. Habitat effects on the relative importance of trait- and density-mediated indirect interactions. Ecology Letters 9: $1245-1252$.

Trussell, G. C., and M. O. Nicklin. 2002. Cue sensitivity, inducible defense, and trade-offs in a marine snail. Ecology 83:1635-1647.

Trussell, G. C., and L. D. Smith. 2000. Induced defenses in response to an invading crab predator: an explanation of historical and geographic phenotypic change. Proceedings of the National Academy of Sciences (USA) 97:2123-2127.

Van Buskirk, J. 2002. A comparative test of the adaptive plasticity hypothesis: relationship between habitat and phenotype in anuran larvae. American Naturalist 160:87102.

Van Buskirk, J., and M. Arioli. 2002. Dosage response of an induced defense: How sensitive are tadpoles to predation risk? Ecology 83:1580-1585.

Van Buskirk, J., and R. A. Relyea. 1998. Selection for phenotypic plasticity in Rana sylvatica tadpoles. Biological Journal of the Linnean Society 65:301-328.

Van Buskirk, J., and B. R. Schmidt. 2000. Predator-induced phenotypic plasticity in larval newts: trade-offs, selection, and variation in nature. Ecology 81:3009-3028.

Van der Stap, I., M. Vos, A. M. Verschoor, N. R. Helmsing, and W. M. Mooij. 2007. Induced defenses in herbivores and plants differentially modulate a trophic cascade. Ecology 88: 2474-2481.

Via, S., and R. Lande. 1985. Genotype-environment interaction and the evolution of phenotypic plasticity. Evolution 39: $505-522$.

Werner, E. E., and S. D. Peacor. 2003. A review of traitmediated indirect interactions in ecological communities. Ecology 84:1083-1100.

Wiackowski, K., and A. Staronska. 1999. The effect of predator and prey density on the induced defence of a ciliate. Functional Ecology 13:59-65.

Wilbur, H. M., and J. E. Fauth. 1990. Experimental aquatic food webs: interactions between two predators and two prey. American Naturalist 135:176-204.

Wootton, J. T. 1994. Putting the pieces together: testing the independence of interactions among organisms. Ecology 75 : $1544-1551$.

\section{APPENDIX}

The data correction method used to exclude the possible effect of selective predation on tadpoles having low bulginess induction (Ecological Archives E090-078-A1). 